\title{
A Quality Assurance Scheme of Higher Engineering Education Accreditation Based on Expert Opinions Integration
}

\author{
Yujie Feng ${ }^{1, a}$, Bin Duan ${ }^{2, b}$ and Xiaoxiao Guo ${ }^{2, c}$ \\ ${ }^{1}$ School of of Information Engineering, Xiangtan University, Xiangtan 411105, China; \\ ${ }^{2}$ Cooperative Innovation Center of Wind Power Equipment and Energy ConversionSchool, \\ XiangtanG 510000, China \\ afengyujieL@163.com, bduanbin@xtu.edu.cn, 'cguo92102@163.com
}

Keywords: Coordinated decision making mechanism; Expert opinions integration; Knowledge automation; Machine learning; Teaching evaluation.

\begin{abstract}
With the constantly advancing of university engineering education accreditation, in the curricular of assessment of teaching quality, establishing an effective quality guarantee system that meets the standard of engineering education accreditation becomes one of issues that need to be addressed urgently in higher education reform. The paper proposes a new Higher Engineering Education Accreditation evaluation scheme to investigate the issue of teaching evaluation in higher education where decision management cycle makes a decision based on integrated expert opinions and generated knowledge in which machine learns, analyzes huge chunks of education data and makes predictions. A collaboration decision mechanism is implemented to assist to make multi-target cooperative decisions for evaluation of teaching, which provides more high-quality expert opinions for teaching. A practical case study has been successfully validated for the adaptability and practicability of the quality assurance scheme where Bayesian techniques process many variables and large of observations to achieve expert opinions integration. The scheme reached a degree of predictive ability, generalization ability, and self-improvement and it can improve the efficiency of teaching management decision.
\end{abstract}

\section{Introduction}

Greater attention has been paid to the quality of research work of Higher Engineering Education Accreditation which China is developing its own accreditation system with the goal of establishing a quality assurance system and promoting the international recognition for engineering education[1][2]. The teaching evaluation is based on general indicators which reflect a simplification of a complex phenomenon; scientific research related to teaching evaluation in higher education is presented and discussed. Quality expert opinion has a great impact on the teaching evaluation; effective quality assurance system needs to integrate expert opinion and social impact of the multi-objective coordination to reach decisions on teaching evaluation.

Teaching evaluation is characterized by multidimensionality and complexity, analysing large amounts of data to support decision-making has caused a lot of industry attention. For data integration, common assessments of domestic and foreign universities use quantitative methods. Several various approaches are developed from data integration[3] [4]:Typically Buhlmann [5] proposed the use of hierarchical reliability theory methods to integrate data, but this method is more sensitive to expert opinion, it does not have a good stability; Analytic Hierarchy Process(AHP) [6] is pairwise comparison to determine the weight of multiple indexes, at the same time,it inevitably avoids subjective judgment. They are unable to resolve the existence of indicators related issues, overlapping information to the results of the evaluation will bring large deviation.

On the basis of the analysis of data integration, the Bayesian inference is applied to education credibility modeling to integrate the external data, internal data and expert opinion, also, to model and estimate the frequency and severity. The scheme can be dynamically updated implementation in Higher Engineering Education Accreditation teaching evaluation based on integrated expert opinions. 


\section{Requirement Analysis And System Architecture}

\subsection{Requirement Analysis.}

According to the major standard certification assessment, combined with the views of the Ministry of education evaluation experts. As Fig1 show, the establishment of an effective quality assurance system is very important, to strengthen engineering education industry, business contacts and cooperation, and to promote international certification engineering education. Teaching Decision Support System provides effective support for Macro College. Teaching Decision includes integrating teaching data, and its comprehensive analysis and processing[7].

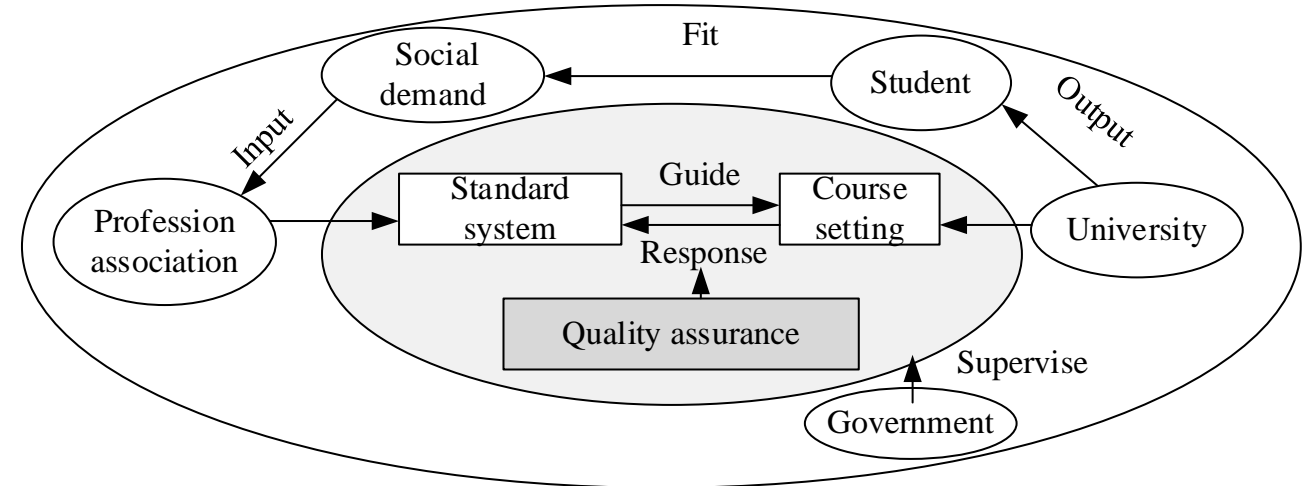

Fig. 1 Conceptual model of major accreditation system

\subsection{Quality assurance system.}

The quality assurance system has the ability of independent decision-making analysis, to solve evaluation of curricular without subjectivity and inconsistency, to analyze coure impact factor and offer degree curriculars to reach decision-making objectively.

\begin{tabular}{|c|}
\hline Extracting the related edcation data \\
\hline $\begin{array}{c}\text { Utilizing collaboration decision mechanism, users rewrite or pass the decision } \\
\text { which is proposed by the curricular achievement degree decision services } \\
\text { according to the third party evaluation }\end{array}$ \\
curricular achievement degree \\
Using the machine learning, obtaining the related impact factor of the \\
Packaging the analyzing model which is formed by impact factor and \\
integrated expert knowledge into the same knowledge base for the recall of the \\
curricular achievement degree decision services \\
Adopting the decision management cycle architecture, using the secondary \\
mining and knowledge discovery for the assessment result, making decisions \\
for the curricular achievement degree by Recycling
\end{tabular}

Fig. 2 Diagram of decision management cycle Architecture

Combined with major certification requirements, as Fig. 2 shows, the quality assurance system requirements include the following:

(1) Teaching-related data includes questions achievements, achievement assessment program, curriculum support group score and businesses, student questionnaire survey ratings, etc. And achieving files informatics.

(2) Data preprocessing is the key to providing high quality data for university education decision support system. The idea of design data preprocessing to provide appropriate data for different education decision tasks, which covers the data quality improvement and data reorganization according to different education themes by data extraction, integration and reduction. 
(3) Expert knowledge according to the needs of experts can make specific adjustments,and the case for decision analysis model used to predict the etermination of the data item which must be the same used for modeling

(4) Secondary mining and knowledge discovery, can enhance the user understandability, and try to identify the potential patterns and rules in order to enhance the accuracy of the results generated mining and practicality.

\section{Decision Management Cycle}

Teaching system, as an open system, needs to mobilize information exchange with the outside world actively, and to find some ways to adapt to change and maintain the viability of the system itself. The Fig. 3 shows the diagram of decision management cycle architecture where associated data knowledge discoveried, data in the database for data mining and pattern discovery implicit rule.To ensure their effectiveness, all knowledge which in the form of impressions of the decision tree must through the use of real data to test. The decision-making will use a large number of historical education data as input and generate a model of knowledge representation as output.

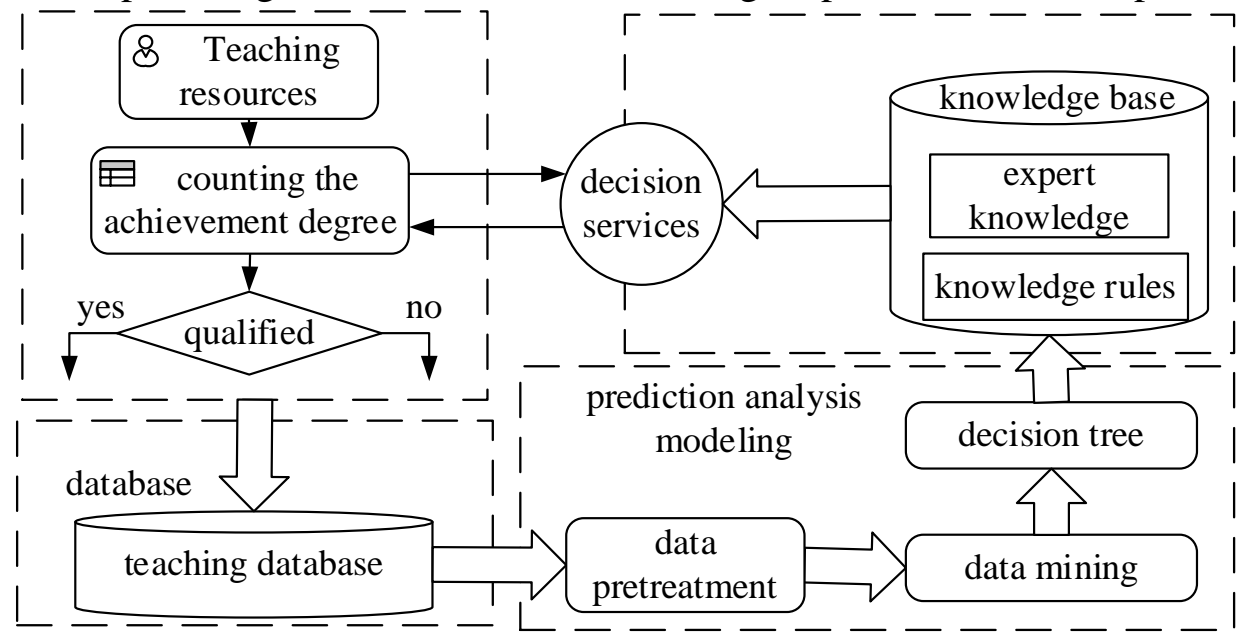

Fig. 3 Diagram of decision management cycle Architecture

\section{Integration Expert Opinion}

The purpose of the integration of expert opinion is to find a method of forming of consensus, which is in order to find the optimal solution or satisfactory solution groups.Thus, the integrated expert opinion can refine your search to achieve.On the basis of original an one-time given expert opinion, the paper builds the reliability of measurement methods expert opinion considering the coherence and coordination factors.Establishment of expert opinions integrated search optimization model, using the information processing power of computers to solve, to get both to meet the consistency requirements, and the level of coordination and credible indicators conclusion.

\section{1 collaboration decision mechanism}

According to the decision-making environment and target problem, considering the model evaluation, between the various indicators, contradiction or conflict may exist, the establishment of comprehensive assessment methods of data mining methods for performance evaluation of the model deep mining. In order to enhance the credibility of the assessment results, choosing a multi-objective decision-making method, can also select multiple multi-objective decision making methods evaluated simultaneously.

There are many factors which can influence collaboration decision design of teaching evaluation[8]. The system chart of curricular achievement degree evaluation with collaboration decision mechanism is seen in Fig. 4. 


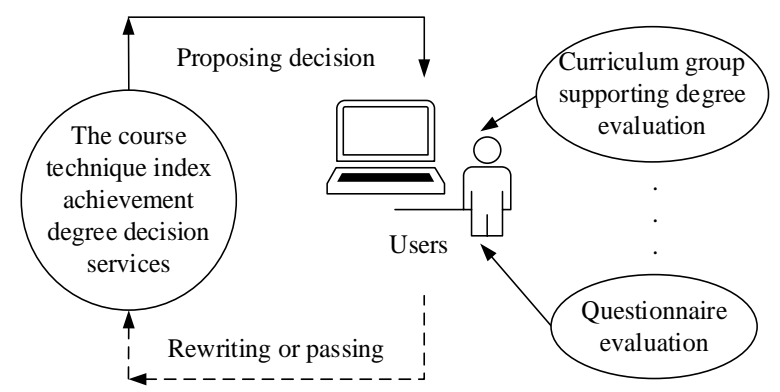

Fig. 4 Chart of curricular achievement evaluation with collaboration decision mechanism

\section{Case Study}

\subsection{Bayesian Analysis.}

Bayesian analysis provides a resource for the further development of new models and statistical methods of teaching evaluation analysis[9]. Bayesian formula predicts the training samples which is calculated from the value of each attribute category as the frequency ratio of the prior probability $P\left(\omega_{1}\right)$ and assumes among individual properties are independent of each other.

$$
\begin{aligned}
& P\left(\omega_{1}\right)=\frac{N_{1}}{N} \\
& P\left(\omega_{2}\right)=\frac{N_{2}}{N}
\end{aligned}
$$

$N_{1}$ is the Number of adopted; $N_{2}$ the Number of failed.

Bayesian conditional probability formula and related probabilities calculates for each instance of the value, and selectes one of the greatest probability value category as the predicted value.

\subsection{Case Background.}

$$
P\left(\omega_{i} \mid \mathrm{X}\right)=\frac{P\left(\omega_{i} \mid \mathrm{X}\right) P\left(\omega_{i}\right)}{\sum_{j=1}^{M} P\left(\omega_{j} \mid \mathrm{X}\right) P\left(\omega_{j}\right)}
$$

Experts are invited to speculate 20 achievements of the curricular accreditation evaluation. Expert A speculates correct 14 times, 10 times the correct B expert speculates, C experts speculates correctly 12 times. Monte Carlo simulation is implemented to perform a risk assessment evaluation by @risk which Simulated 5000 times.Table 1shows the expert background.

Table 1 Expert Background

\begin{tabular}{|c|c|c|c|c|c|c|}
\hline & $\begin{array}{c}\text { Educational } \\
\text { Background }\end{array}$ & Title & Seniority & Accuracy(\%) & $\begin{array}{c}\text { Total } \\
\text { Score }\end{array}$ & Weight \\
\hline A & Doctor & Professor & 25 & 72.7 & 117.7 & 0.4 \\
\hline B & Doctor & Associate Professor & 20 & 50.0 & 88.0 & 0.3 \\
\hline C & Master & Associate Professor & 15 & 59.1 & 90.1 & 0.3 \\
\hline
\end{tabular}

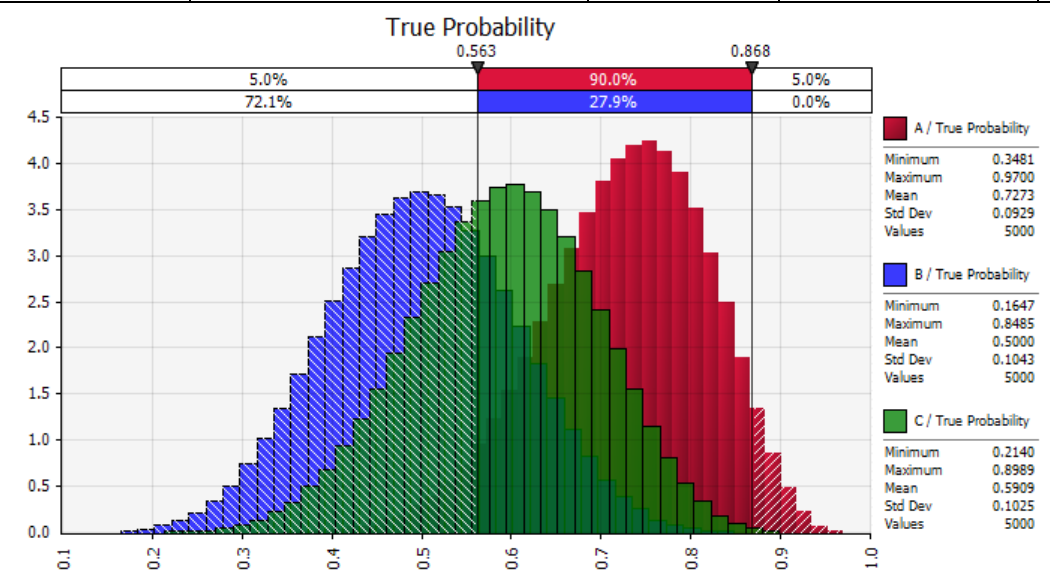

Fig. 5 The true probality of speculated accuracy 
Bayesian inference analysis obtained true probability of speculated accuracy as shown in Fig. 5. combined with background data, the respective feasible weight of the expert $\mathrm{A}, \mathrm{B}$ and $\mathrm{C}$ are $0.4,0.3$ and 0.3 .

\subsection{Integration Expert Opinion.}

The reliability of expert opinion is a specialist in the distribution of a given distribution function within the range of opinion scores, which function satisfies the continuous, bounded, symmetric, monotonic segments of different characteristics. Reliability different expert opinions can be considered independent and identically distribution. If the three experts were designated variable is PERT distribution parameters as shown in Table. 2

Table 2 Experts predicted value analysis table

\begin{tabular}{|c|c|c|c|c|c|}
\hline & $\begin{array}{l}\text { minimum } \\
\text { value }\end{array}$ & $\begin{array}{c}\text { probable } \\
\text { value }\end{array}$ & $\begin{array}{l}\text { maximum } \\
\text { value }\end{array}$ & weight & distribution \\
\hline A & 45 & 50 & 70 & 0.4 & 52.50 \\
\hline B & 50 & 65 & 90 & 0.3 & 66.67 \\
\hline $\mathrm{C}$ & 50 & 65 & 80 & 0.3 & 65.00 \\
\hline integrated & & & & & 65.00 \\
\hline
\end{tabular}

Expert opinion on the amount of the adjustment is not allowed beyond the experts given allowable adjustment range. Final prediction distributed as Fig. 6 shown. Various expert opinions can be integrated and coordinated efficiently, and gives reliability index, increased integration of the results of that convincing and credible degree.

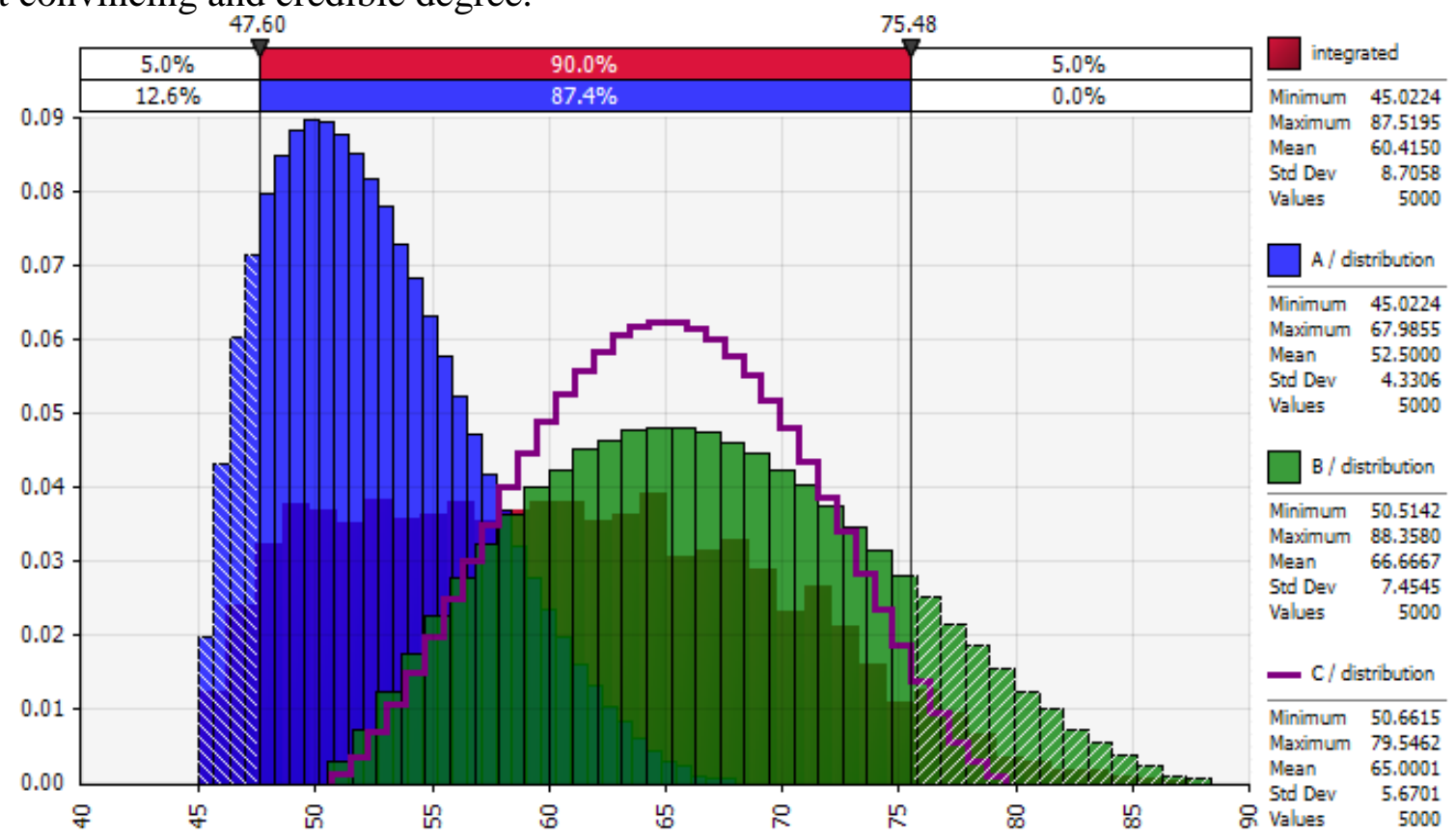

Fig. 6 Expert opinion prediction distributed

Experts Opinion integration is not only theoretically feasible, but also in the use of expert advice can reduce the number of repeated adjustments. The original expert opinion is based on analysis of data obtained by the result of the integration, to ensure the independence of expert advice, so that the original information of the expert analysis of the problem of judgment and perception is preserved, each expert opinions have been the greatest degree of respect.

\section{Conclusion}

Through comprehensively considering evidence certainty factor and average degree support of subset in frame of discernment, the paper efficiently solves the phenomenon of one veto existing in integration of highly conflict opinion, accurately reflects the assessment opinion of expert and improving the reliability of the whole system. 
In the context of engineering education accreditation, evaluating the achievement degree in just and fair environment. The paper provides a quality assurance scheme into the China specific educational situation within expert opinions integration, which promotes international recognition for engineering education. This study aims to identify the effects of evaluation on teaching and discusses improvements in the work of the evaluation office and the progress and potential impact of building engineering education accreditation system in its initial period. Faster feedback and higher quality comments are perceived to provide more help to engineering education and the industry.

\section{Acknowledgment}

This research is supported by National Natural Science Foundation of China (NSFC) (No.61170191, 61379063). The authors would like to thank for the Collaborative Innovation Center of wind power equipment and energy conversion.

\section{References}

[1]. WX. Zhang, "Development of Engineering Education Accreditation System and Its Potential Impact on Higher Engineering Education”, Tsinghua Journal of EducationT, 2011, pp.24-26.

[2]. WS. Chen, "Engineering Education Major Certification and Its Influence on Higher Engineering Education”, Higher Education Forum, 2011, pp. 86-90.

[3]. Lenzerini and Maurizio, "Data Integration: A Theoretical Perspective." Symposium on Principles of Database Systems, 2015, pp. 233-246.

[4]. Y. Peng, "An incident information management framework based on data integration, data mining, and multi-criteria decision making." Decision Support Systems vol. 2, 2011, pp. 316-327.

[5]. Peter Fratzl, and Richard Weinkamer. "Nature’s hierarchical materials." Progress in Materials Science vol. 52(52), 2007, pp. 1263-1334.

[6]. Ishizaka, Alessio, and P. Nemery, Analytic hierarchy process. Encyclopedia of Biostatistics, 2005.

[7]. S.Vinnik, M.H.Sholl, and UNICAP, "Efficient decision support for academic resource and capacity anagement", International Federation for Information Processing, 2005. pp. 235-246.

[8]. Fish, Alan N, and J. Taylor, Knowledge Automation, 2012.

[9]. Jacquler, N. G. Polson, and P. E. Rossi, "Analysis of Stochastic Volatility Models." Journal of Business \& Economic Statistics vol.12(1), 2012, pp. 371-89. 